\title{
Ballistic Graphene Cooper Pair Splitter
}

\author{
P. Pandey, ${ }^{1}$ R. Danneau $\odot,{ }^{2, *}$ and D. Beckmann $\odot^{2, \dagger}$ \\ ${ }^{1}$ Institute of Nanotechnology, Karlsruhe Institute of Technology, Karlsruhe D-76021, Germany \\ ${ }^{2}$ Institute for Quantum Materials and Technologies, Karlsruhe Institute of Technology, Karlsruhe D-76021, Germany
}

(Received 19 May 2020; accepted 5 March 2021; published 7 April 2021)

\begin{abstract}
We report an experimental study of a Cooper pair splitter based on ballistic graphene multiterminal junctions. In a two transverse junction geometry, namely the superconductor-graphene-superconductor and the normal metal-graphene-normal metal, we observe clear signatures of Cooper pair splitting in the local as well as nonlocal electronic transport measurements. Our experimental data can be very well described by our beam splitter model. These results open up possibilities to design new entangled state detection experiments using ballistic Cooper pair splitters.
\end{abstract}

DOI: 10.1103/PhysRevLett.126.147701

Controlling entanglement of quantum states is at the heart of quantum computing and quantum information [1]. However, the generation, detection, and manipulation of entangled quantum states remain a serious challenge in scalable solid state systems as the interactions and excitations are detrimental [2-4]. Cooper pair splitting (CPS) [5-15] provides efficient electron spin-entangled sources and, therefore, could possibly be used in on-chip quantum state teleportation experiments [16]. The splitting of Cooper pairs refers to the time reversal phenomenon of nonlocal Andreev reflection (also called crossed-Andreev reflection) [17-31] and has been observed when a superconductor is coupled to multiterminal systems such as InAs nanowires [5,8,9], carbon nanotubes [6,10,11], normal metal-insulator-superconductor junctions [7], and etched graphene based quantum dots $[12,14,15]$. While various applications of CPS have been proposed [32-36], a demonstration of entanglement is still missing. Entanglement can be probed, for example, by measuring noise correlations in beam splitter geometries [37-39]. Here, we show the experimental observation of CPS in a ballistic graphene beam splitter, which opens a path toward entanglement detection in more complex geometries.

Graphene-superconductor interfaces exhibit very peculiar Andreev processes at low energy [40-45], and nonlocal processes have been predicted to occur under various conditions [46-48], including in the quantum Hall regime [28-31]. Thanks to the progress in device fabrication [49], graphene appears to be a clean and highly tunable two-dimensional material that couples very well to superconductors where a ballistic effect can be directly observed [50-58]. However, to our knowledge, very limited studies on nonlocal processes in clean graphene have been reported until now $[59,60]$.

Our device consists of single layer graphene encapsulated in hexagonal boron nitride (h-BN). The graphene sheet is connected to two superconductors on its parallel edges and to two normal metal electrodes on the other two transverse edges. As a result, two transverse junctions are formed, namely the superconductor-graphene-superconductor (SGS) and the normal metal-graphene-normal metal (NGN) junctions. Because of a difference in the doping density across the two junctions, potential barriers are generated in the graphene channel that act as electronic beam splitters for CPS similar to the one proposed by Bouchiat et al. [22] for the case of a single walled carbon nanotube. We employ a modified Octavio-Tinkham-Blonder-Klapwijk (OTBK) model [57,61-64] along with a three-terminal beam splitter model to explain our experimental observation. The advantage of this implementation is that the Andreev reflection takes place locally at a single interface and is not limited by contact spacing.

We have used the dry transfer technique for the fabrication of h-BN-graphene-h-BN van der Waals heterostructure similar to the one described in [49]. Edge contacts to the encapsulated graphene layer were established in a self-aligned manner as described in [54] and adapted for two different contact materials in [57]. Details of the devices are shown in the Supplemental Material [65]. The device schematics are shown in Figs. 1(a) and 1(b), and the false color atomic force micrograph is shown in Fig. 1(c).

As the device consists of two perpendicular junctions with two different contact metals, we first characterize the device in the normal state, i.e., above the critical temperature $\left(T_{c} \approx 1 \mathrm{~K}\right)$ of the superconductor, as it helps in understanding the electronic transport across the graphene channel connected by two different normal metals. Figures 2(a) and 2(b) show the gate dependent resistance of the device across the SGS and NGN junctions, respectively, in the normal state at $T=6 \mathrm{~K}$ [66]. We observe that the charge neutrality point (CNP) is shifted to the negative gate voltages for both of the junctions, which indicates n-type doping of the graphene sheet. This can be 


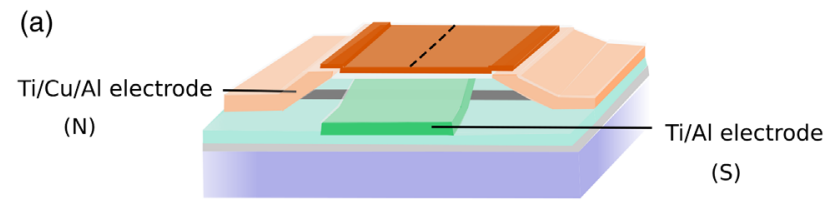

(b)

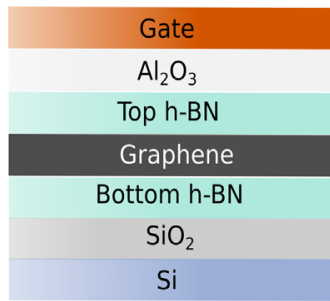

(c)

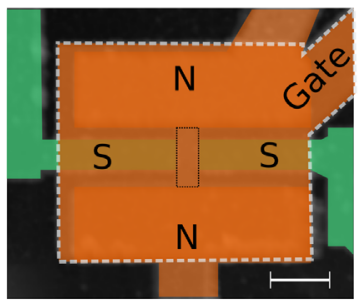

FIG. 1. (a) Schematic of the device geometry. (b) Cross section of the device across the dashed line shown in (a). (c) False color atomic force micrograph of the studied device showing the normal (N) and superconducting (S) electrodes. Scale bar is $1 \mu \mathrm{m}$. The area enclosed by the white dashed lines shows the top gate electrode. The black dotted line shows roughly the h-BN encapsulated graphene.

explained by the charge transfer from metal contacts forming a potential barrier when the Fermi level of graphene is tuned in the valence band [67-69]. Since graphene is doped n-type by the metal contacts, pn junctions form in the vicinity of the graphene-metal interface when the Fermi level is driven into the holedoped regime. As a result, an electronic Fabry-Pérot (FP) cavity is formed, which manifests itself in the form of periodic oscillations in the conductance or resistance of graphene, known as the FP resonances, when the charge transport is in the ballistic regime. These resonances can be clearly observed in the p-doped region in the SGS junction, as shown in the inset in Fig. 2(a) (see the Supplemental Material [65] for a detailed analysis). The presence of FP resonances clearly indicates that the charge transport in the SGS junction is in the ballistic regime. Importantly, the two junctions have different doping densities as the CNP for the SGS junction is at the gate voltage $V_{g}=-2.6 \mathrm{~V}$, while the CNP for the NGN junction is at $V_{g}=-1.8 \mathrm{~V}$. The difference in the two CNPs indicates that the SGS junction is heavily n-doped as compared to the NGN junction. Since the transport across the NGN junction includes the contribution from the SGS junction, the doping profile along the NGN junction consists of three different regions, as shown in Figs. 2(c) and 2(e) and explained in the Supplemental Material [65]. In this case, the central graphene region has a different doping density than the two outer regions, and this suggests that, apart from the potential barriers at the metal-graphene interface, there are additional potential barriers along the length of the graphene sheet across the NGN junction [70].

After the normal state measurement, we study the Andreev processes at millikelvin temperatures. For
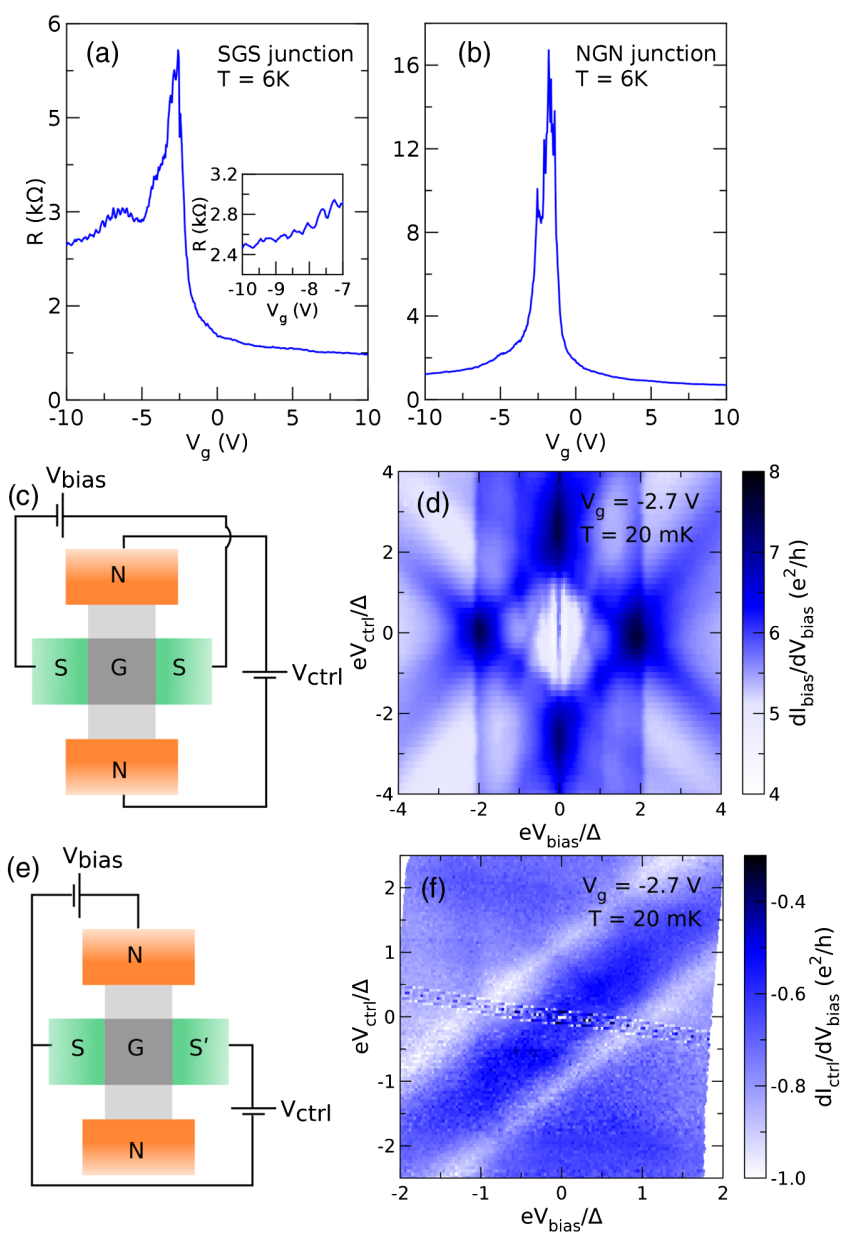

FIG. 2. Gate dependent resistance of the device across the (a) SGS junction (inset: FP resonances in the p-doped regime) and (b) NGN junction in the normal state. (c) Four-terminal or SGS configuration. (d) Local differential conductance $d I_{\text {bias }} / d V_{\text {bias }}$ map across the SGS junction in the superconducting state at $V_{g}=-2.7 \mathrm{~V}$ and $T=20 \mathrm{mK}$ under the zero magnetic field in the SGS configuration. (e) Three-terminal or NGS configuration. (f) Nonlocal differential conductance $d I_{\text {Ctrl }} / d V_{\text {bias }}$ map across the SGS junction in the superconducting state at $V_{g}=-2.7 \mathrm{~V}$ and $T=20 \mathrm{mK}$ at zero magnetic field in the NGS configuration.

this purpose, we employ two different measurement configurations, namely the four-terminal or SGS configuration and the three-terminal or normal metal-graphene-superconductor (NGS) configuration. Figure 2(c) shows the SGS configuration where the bias voltage $V_{\text {bias }}$ is applied across the SGS junction, while the control voltage $V_{\text {Ctrl }}$ is applied across the NGN junction. Figure 2(d) shows the local differential conductance $d I_{\text {bias }} / d V_{\text {bias }}$ map across the SGS junction obtained in the four-terminal configuration at $V_{g}=-2.7 \mathrm{~V}$ (close to the CNP of the SGS junction) and $T=20 \mathrm{mK}$ under the zero magnetic field. Note that the $V_{\text {bias }}$ and $V_{\text {Ctrl }}$ are normalized with the superconducting gap $\Delta$ where $\Delta=130 \mu \mathrm{eV}$ as observed in the experiments. The vertical features appearing at $e V_{\text {bias }} / \Delta= \pm 1$ and \pm 2 
can be assigned to the multiple Andreev reflections (MAR) in the SGS junction as they appear to be independent of $V_{\text {Ctrl }}$. However, there are additional conductance features that can be clearly observed in the map. First is the split diamondlike pattern that can be observed throughout the entire measurement range. Second is the distinct crosslike features that can be observed in the region $\left|e V_{\text {bias }} / \Delta\right| \leq 1$ and $\left|e V_{\mathrm{Ctrl}} / \Delta\right| \leq 2$, and third, a vertical conductance ridge at $\left|e V_{\text {bias }} / \Delta\right|=0$ when $\left|e V_{\mathrm{Ctrl}} / \Delta\right| \geq 2$. All of these features are tuned by the $V_{\text {bias }}$ and $V_{\mathrm{Ctrl}}$. Given our device multiterminal geometry, it suggests that there could be other Andreev processes taking place in the system [71,72]. Next, we employ the three-terminal configuration [see Fig. 2(e)], where $V_{\text {bias }}$ is applied across one of the NGS junctions and $V_{\text {Ctrl }}$ is applied across the SGS junction. The $\mathrm{S}$ terminal, which is outside the bias circuit and involved only in the control circuit, is labeled $S^{\prime}$ for the sake of clarity. We measure the nonlocal differential conductance $d I_{\text {Ctrl }} / d V_{\text {bias }}$ across the SGS junction under the same conditions as for the data shown in Fig. 2(d). The resulting $d I_{\text {Crrl }} / d V_{\text {bias }}$ map is shown in Fig. 2(f). We observe nearly vertical features that could be assigned to the direct transport in the bias circuit and nearly horizontal features that could come from the direct transport in the control circuit. Similar to the data shown in Fig. 2(d), there are other clearly observable conductance features that are influenced by the $V_{\text {bias }}$ and $V_{\text {Ctrl }}$.

In order to interpret the experimental data, we employ a modified OTBK model [57,61-64] for the transport between each pair of terminals, as indicated by the solid lines in Fig. 3(a). To account for nonlocal transport processes, we include a beam splitter model in addition to the two-terminal contributions. The beam splitters in our device are expected to form due to interfaces between regions of different doping densities where Klein tunneling leads to an angle-dependent partial transmission and reflection [42,73]. Given our device geometry, nonlocal Andreev processes that involve both of the $S$ terminals and one $\mathrm{N}$ terminal are most likely to take place. From the pairwise two-terminal resistances measured in the normal state, we know that the device is highly asymmetric with respect to the four corners, especially near the CNP. To incorporate nonlocal processes and the asymmetry of the device at the same time, we model four beam splitters that follow the four corners of the device, as indicated by dashed lines in Fig. 3(a). As an example, the beam splitter in the top left corner is shown in detail in Fig. 3(b). To follow the corner, an electron from the top $\mathrm{N}$ terminal can be transmitted only to the left $S$ terminal, with probability $\tau_{\mathrm{tl}}$. An electron from the right $S$ terminal can be transmitted with probability $\tau_{\mathrm{lr}}=1-\tau_{\mathrm{tl}}$ to the left terminal. For simplicity, we assume that there is no reflection back to the left terminal. It is to be noted that the beam splitters also contribute to the local processes between the NS and SS terminals. Therefore, the model also includes nonlocal (a)
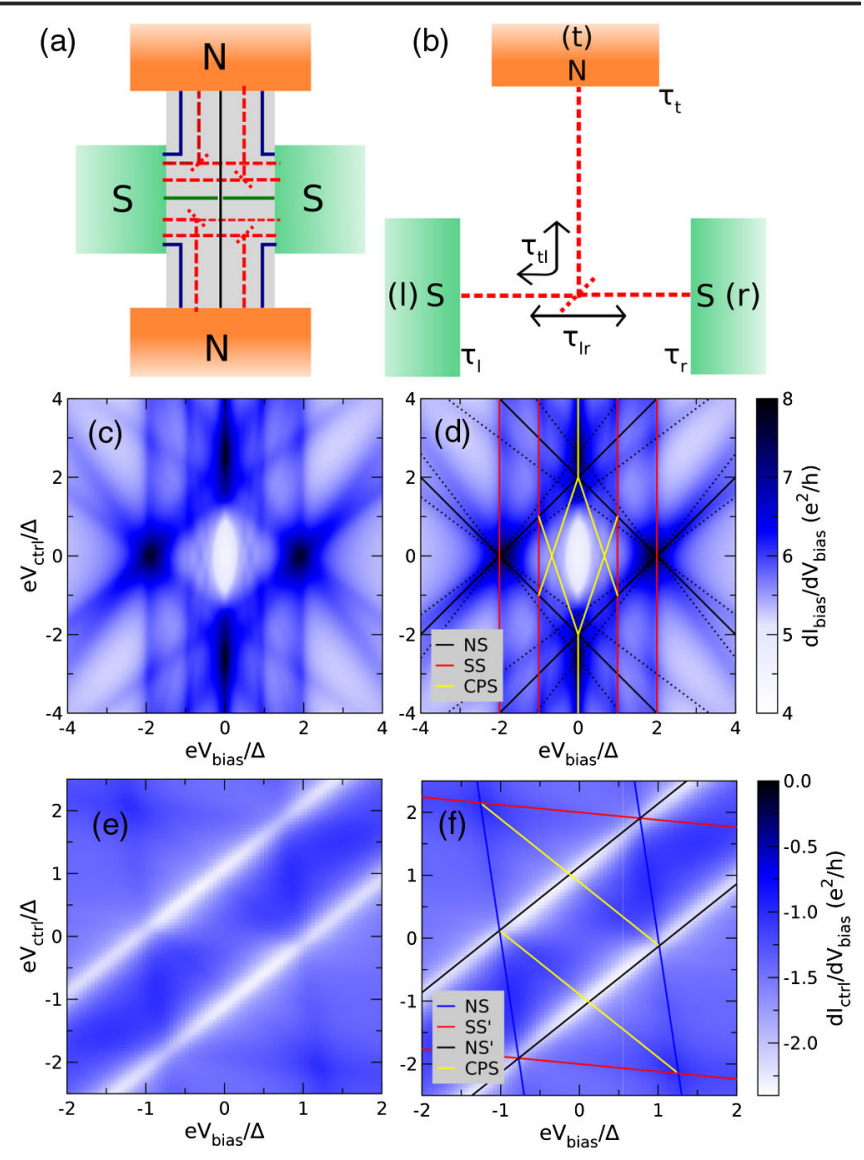

FIG. 3. (a) Schematics of the model showing local (solid) and nonlocal (dashed) transport channels. (b) Three-terminal beam splitter for the nonlocal transport channels as shown in (a) (t, l, and $\mathrm{r}$ stand for top, left, and right, respectively). Differential conductance maps generated with the model: local differential conductance $d I_{\text {bias }} / d V_{\text {bias }}$ map in the SGS configuration without (c) and with (d) the guidelines, and nonlocal differential conductance $d I_{\text {Crrl }} / d V_{\text {bias }}$ map in the NGS configuration without (e) and with (f) the guidelines.

MAR processes where an initial nonlocal Andreev process starts a MAR cycle between the two superconductors (see the Supplemental Material [65] for details).

The result generated with the model and corresponding to the experimental data in Fig. 2(d) (SGS configuration) is shown in Fig. 3(c), including guidelines for interpretation in Fig. 3(d). The interpretation is based on the bias thresholds for different transport processes. In particular, the conditions for Cooper pair splitting are shown in Fig. 4 and explained in detail below. The transmission coefficients at the superconducting terminals were chosen to be 0.72 , while they were 0.7 at the normal metal terminals. Comparing Fig. 2(d) to Fig. 3(c), it can be readily seen that the experimental data and the model are in very good agreement. To interpret the observed conductance features, we compare Fig. 2(d) to Fig. 3(d). The vertical conductance features at $e V_{\text {bias }} / \Delta= \pm 1$ and \pm 2 [marked SS in Fig. 3(d)] appear due to the direct transport between the two 


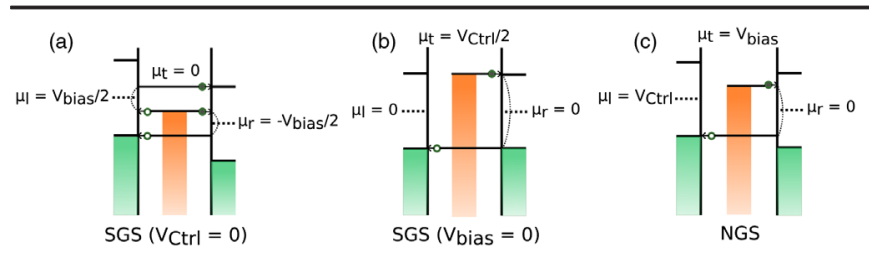

FIG. 4. Bias conditions for the observation of nonlocal Andreev processes in the SGS configuration at (a) $V_{\mathrm{Ctrl}}=0$ and (b) $V_{\text {bias }}=0$ and (c) in the NGS configuration. $\mu$ represents the chemical potential of the respective terminal. Filled and empty circles denote the electrons and holes, respectively.

superconductors. The diamondlike feature [marked NS in Fig. 3(d)] appears because of the transport across the NGS corners of the device. The splitting spread of this feature results from the asymmetry of the corner contacts. Most interesting are the crosslike features that appear in the region $\left|e V_{\text {bias }} / \Delta\right| \leq 1$ and $\left|e V_{\mathrm{Ctrl}} / \Delta\right| \leq 2$ and the vertical conductance ridge at $\left|e V_{\text {bias }} / \Delta\right|=0$ when $\left|e V_{\text {Ctrl }} / \Delta\right| \geq 2$ [marked CPS in Fig. 3(d)]. These features appear as a result of the nonlocal transport involving the $\mathrm{N}$ and $\mathrm{S}$ terminals. While the crosslike features can be unambiguously assigned to the Cooper pair splitting, the vertical conductance ridge remains slightly ambiguous due to the possibility of a very weak supercurrent flowing through the SGS junction. We note that the measurements were conducted close to the CNP of the SGS junction where the resistance of the graphene channel is very high, as shown in Fig. 2(a). Therefore, the supercurrent magnitude is too small to be measured. However, its contribution cannot be completely ruled out (see the Supplemental Material [65] for additional gate voltage measurements). It is noteworthy that the conductance features appear in a low bias regime, and as $V_{g}$ is tuned far away from the CNP, the supercurrent contributions as well as the increased conductance of the graphene channel start to overshadow the CPS features.

Figure 3(e) shows the $d I_{\text {Ctrl }} / d V_{\text {bias }}$ map for the NGS configuration as generated with the model, which corresponds to the experimental data shown in Fig. 2(f), while Fig. 3(f) shows the same map but with the guidelines. Comparing Fig. 2(f) to Fig. 3(e), we can see that there is a qualitative agreement between the experimental data and the model. It is to be noted that the experimental measurement in the NGS configuration was two-probe instead of the pseudo-four-probe as in the SGS configuration. As a result, the experimental data includes the series resistances from the filters in the measurement lines. Therefore, the comparison between the experimental data and the model in the NGS configuration is only qualitative. Figures 2(f) and 3(f) can be compared for the interpretation of the various conductance features. There are three different sets of features that appear due to the direct transport in the device, namely the nearly vertical ones due to the transport across the bias junction [marked NS in Fig. 3(f)], the nearly horizontal features due to the transport across the control junction [marked $\mathrm{SS}^{\prime}$ in Fig. 3(f)], and the features with negative contribution that appear due to the transport across the $\mathrm{NGS}^{\prime}$ junction [marked $\mathrm{NS}^{\prime}$ in Fig. 3(f)]. In this case, too, we clearly observe the conductance features [marked CPS in Fig. 3(f)], which appear only due to the nonlocal Andreev process in the device.

As seen in Figs. 2(d) and 2(f), the nonlocal Andreev processes appear in a certain $V_{\text {bias }}$ and $V_{\text {Ctrl }}$ range. The onset of these processes can be explained by considering the energy thresholds. For the SGS configuration at $V_{\text {Ctrl }}=0$, the threshold for the onset of nonlocal Andreev process is at $e V_{\text {bias }}=2 \Delta / 3$ as shown in Fig. 4(a). In this case, an electron that incidents from the normal metal is Andreev reflected at the left superconductor, while the resulting hole enters the right superconductor. On the other hand, for a hole that incidents from the normal metal onto the right superconductor, the Andreev reflected electron enters the left superconductor. As soon as $\left|V_{\text {Ctrl }}\right|>0$, the threshold for $V_{\text {bias }}$ changes and this results in the cross-shaped feature observed in Fig. 2(d) in the regions $\left|e V_{\text {bias }} / \Delta\right| \leq 1$ and $\left|e V_{\text {Crrl }} / \Delta\right| \leq 2$. For the condition $V_{\text {bias }}=0$ in the SGS configuration, the nonlocal Andreev reflection is enabled as soon as $e V_{\text {Ctrl }} \geq 2 \Delta$, as illustrated in Fig. 4(b). This is seen as the vertical conductance ridge in Fig. 2(d) in the region $\left|e V_{\text {bias }} / \Delta\right|=0$ when $\left|e V_{\mathrm{Ctrl}} / \Delta\right| \geq 2$. Because of a peak in the Andreev reflection probability at $\epsilon=\Delta$, a conductance peak is observed when the gap features of the two superconductors are aligned with each other. In the NGS configuration, the nonlocal Andreev process is enabled when $e V_{\text {bias }}+e V_{\text {Ctrl }}=\Delta$, as shown in Fig. 4(c), and results in the conductance feature observed in Fig. 2(f).

Our model is agnostic to the actual implementation of the beam splitters in the device. Possible candidates are (1) pn junctions between different doping levels across the NGN and SGS junction as evidenced by the different positions of the CNPs in Figs. 2(a) and 2(b), (2) the pn junctions near the superconducting interfaces that give rise to the FP cavity, and (3) more complicated doping inhomogeneities near the CNP as evidenced by the conductance asymmetry in the normal state (see also the Supplemental Material [65]). Our conclusions about the bias thresholds for CPS are independent, however, of this detail.

To conclude, we have observed CPS in a multiterminal graphene device in the ballistic regime. Our device takes advantage of its simple geometry with two different transverse junctions and a tunable doping profile across the graphene channel to split the Cooper pair. Furthermore, the CPS signature is clearly observed in the local as well as nonlocal differential conductance measurements. These observations are very well supported by the modified OTBK model and the three-terminal beam splitter model. Our work shows an experimentally accessible way to achieve and control the spatially separated quantum entangled particles in graphene by using the local tuning 
of the Fermi level and the angle-dependent transmission through the potential barriers [74,75]. Furthermore, electronic transport in the graphene channel can be tuned by using additional gates to form controlled potential barriers that will result in an improved efficiency of the beam splitters. Similar studies can be performed by using bilayer graphene where the valley degree of freedom and electrostatically induced band gap can provide additional control parameters [54,76-78].

The authors thank R. Mélin and B. Douçot for fruitful discussions. This work was partly supported by the Helmholtz Society through Program STN and the DFG via the Project No. DA 1280/3-1. P. P. acknowledges support from a Deutscher Akademischer Austauschdienst (DAAD) scholarship.

* Corresponding author. romain.danneau@kit.edu ${ }^{\dagger}$ Corresponding author. detlef.beckmann@kit.edu

[1] M. A. Nielsen and I. L. Chuang, Quantum Computation and Quantum Information (Cambridge University Press, Cambridge, England, 2000).

[2] M. H. Devoret and R. J. Schoelkopf, Superconducting circuits for quantum information: An outlook, Science 339, 1169 (2013).

[3] P. Krantz, M. Kjaergaard, F. Yan, T. P. Orlando, S. Gustavsson, and W. D. Oliver, A quantum engineers guide to superconducting qubits, Appl. Phys. Rev. 6, 021318 (2019).

[4] G. Burkard, M. J. Gullans, X. Mi, and J. R. Petta, Superconductor-semiconductor hybrid-circuit quantum electrodynamics, Nat. Rev. Phys. 2, 129 (2020).

[5] L. Hofstetter, S. Csonka, J. Nygård, and C. Schönenberger, Cooper pair splitter realized in a two-quantum-dot Y-junction, Nature (London) 461, 960 (2009).

[6] L. G. Herrmann, F. Portier, P. Roche, A. L. Yeyati, T. Kontos, and C. Strunk, Carbon Nanotubes as Cooper-Pair Beam Splitters, Phys. Rev. Lett. 104, 026801 (2010).

[7] J. Wei and V. Chandrasekhar, Positive noise crosscorrelation in hybrid superconducting and normal-metal three-terminal devices, Nat. Phys. 6, 494 (2010).

[8] L. Hofstetter, S. Csonka, A. Baumgartner, G. Fülöp, S. d'Hollosy, J. Nygård, and C. Schönenberger, Finite-Bias Cooper Pair Splitting, Phys. Rev. Lett. 107, 136801 (2011).

[9] A. Das, Y. Ronen, M. Heiblum, D. Mahalu, A. V. Kretinin, and H. Shtrikman, High-efficiency Cooper pair splitting demonstrated by two-particle conductance resonance and positive noise cross-correlation, Nat. Commun. 3, 1165 (2012).

[10] J. Schindele, A. Baumgartner, and C. Schönenberger, NearUnity Cooper Pair Splitting Efficiency, Phys. Rev. Lett. 109, 157002 (2012).

[11] J. Schindele, A. Baumgartner, R. Maurand, M. Weiss, and C. Schönenberger, Nonlocal spectroscopy of Andreev bound states, Phys. Rev. B 89, 045422 (2014).

[12] Z. B. Tan, D. Cox, T. Nieminen, P. Lähteenmki, D. Golubev, G. B. Lesovik, and P. J. Hakonen, Cooper pair splitting by means of graphene quantum dots, Phys. Rev. Lett. 114, 096602 (2015).

[13] G. Fülöp, F. Domínguez, S. d'Hollosy, A. Baumgartner, P. Makk, M. H. Madsen, V. A. Guzenko, J. Nygård, C. Schönenberger, A. L. Yeyati, and S. Csonka, Magnetic Field Tuning and Quantum Interference in a Cooper Pair Splitter, Phys. Rev. Lett. 115, 227003 (2015).

[14] I. V. Borzenets, Y. Shimazaki, G. F. Jones, M. F. Craciun, S. Russo, M. Yamamoto, and S. Tarucha, High efficiency CVD graphene-lead $(\mathrm{Pb})$ Cooper pair splitter, Sci. Rep. 6, 23051 (2016).

[15] Z. B. Tan, A. Laitinen, N. S. Kirsanov, A. Galda, V. M. Vinokur, M. Haque, A. Savin, D. S. Golubev, G. B. Lesovik, and P. J. Hakonen, Thermoelectric current in a graphene Cooper pair splitter, Nat. Commun. 12, 138 (2021).

[16] C. H. Bennett, G. Brassard, C. Crpeau, R. Jozsa, A. Peres, and W. K. Wootters, Teleporting an Unknown Quantum State Via Dual Classical and Einstein-Podolsky-Rosen Channels, Phys. Rev. Lett. 70, 1895 (1993).

[17] J. M. Byers and M. E. Flatté, Probing Spatial Correlations with Nanoscale Two-Contact Tunneling, Phys. Rev. Lett. 74, 306 (1995).

[18] G. Deutscher and D. Feinberg, Coupling superconductingferromagnetic point contacts by Andreev reflections, Appl. Phys. Lett. 76, 487 (2000).

[19] G. Falci, D. Feinberg, and F. W. J. Hekking, Correlated tunneling into a superconductor in a multiprobe hybrid structure, Europhys. Lett. 54, 255 (2001).

[20] G. Deutscher, Crossed Andreev reflections, J. Supercond. 15, 43 (2002).

[21] G. B. Lesovik, T. Martin, and G. Blatter, Electronic entanglement in the vicinity of a superconductor, Eur. Phys. J. B. 24, 287 (2001).

[22] V. Bouchiat, N. Chtchelkatchev, D. Feinberg, G. B. Lesovik, T. Martin, and J. Torrés, Single-walled carbon nanotubesuperconductor entangler: Noise correlations and EinsteinPodolsky-Rosen states, Nanotechnology 14, 77 (2003).

[23] A. L. Yeyati, F. S. Bergeret, A. Martín-Rodero, and T. M. Klapwijk, Entangled Andreev pairs and collective excitations in nanoscale superconductors, Nat. Phys. 3, 455 (2007).

[24] D. Beckmann, H. B. Weber, and H. v. Löhneysen, Evidence for Crossed Andreev Reflection in Superconductor-Ferromagnet Hybrid Structures, Phys. Rev. Lett. 93, 197003 (2004).

[25] D. Beckmann and H. v. Löhneysen, Negative four-terminal resistance as a probe of crossed Andreev reflection, Appl. Phys. A 89, 603 (2007).

[26] S. Russo, M. Kroug, T. M. Klapwijk, and A. F. Morpurgo, Experimental Observation of Bias-Dependent Nonlocal Andreev Reflection, Phys. Rev. Lett. 95, 027002 (2005).

[27] P. Cadden-Zimansky and V. Chandrasekhar, Nonlocal Correlations in Normal-Metal Superconducting Systems, Phys. Rev. Lett. 97, 237003 (2006).

[28] D. J. Clarke, J. Alicea, and K. Shtengel, Exotic circuit elements from zero-modes in hybrid superconductorquantum-Hall systems, Nat. Phys. 10, 877 (2014).

[29] Z. Hou, Y. Xing, A.-M. Guo, and Q.-F. Sun, Crossed Andreev effects in two-dimensional quantum Hall systems, Phys. Rev. B 94, 064516 (2016). 
[30] G.-H. Lee, K.-F. Huang, D. K. Efetov, D. S. Wei, S. Hart, T. Taniguchi, K. Watanabe, A. Yacoby, and P. Kim, Inducing superconducting correlation in quantum Hall edge states, Nat. Phys. 13, 693 (2017).

[31] S.-B. Zhang and B. Trauzettel, Perfect Crossed Andreev Reflection in Dirac Hybrid Junctions in the Quantum Hall Regime, Phys. Rev. Lett. 122, 257701 (2019).

[32] C. Benjamin and J. K. Pachos, Detecting entangled states in graphene via crossed Andreev reflection, Phys. Rev. B 78, 235403 (2008).

[33] S. K. F. Islam and A. Saha, Amplification of Cooper pair splitting current in a graphene-based Cooper pair beam splitter geometry, Phys. Rev. B 96, 125406 (2017).

[34] R. Sánchez, P. Burset, and A. L. Yeyati, Cooling by Cooper pair splitting, Phys. Rev. B 98, 241414 (2018).

[35] N. S. Kirsanov, Z. B. Tan, D. S. Golubev, P. J. Hakonen, and G. B. Lesovik, Heat switch and thermoelectric effects based on Cooper-pair splitting and elastic cotunneling, Phys. Rev. B 99, 115127 (2019).

[36] R. Hussein, M. Governale, S. Kohler, W. Belzig, F. Giazotto, and A. Braggio, Braggio, Nonlocal thermoelectricity in a Cooper-pair splitter, Phys. Rev. B 99, 075429 (2019).

[37] G. Burkard, D. Loss, and E. V. Sukhorukov, Noise of entangled electrons: Bunching and antibunching, Phys. Rev. B 61, R16303 (2000).

[38] P. Samuelsson, E. V. Sukhorukov, and M. Büttiker, Electrical current noise of a beamsplitter as a test of spin entanglement, Phys. Rev. B 70, 115330 (2004).

[39] M. Flöser, D. Feinberg, and R. Mélin, Absence of split pairs in cross correlations of a highly transparent normal metalsuperconductor-normal metal electron-beam splitter, Phys. Rev. B 88, 094517 (2013).

[40] C. W. J. Beenakker, Specular Andreev Reflection in Graphene, Phys. Rev. Lett. 97, 067007 (2006).

[41] M. Titov, A. Ossipov, and C. W. J. Beenakker, Excitation gap of a graphene channel with superconducting boundaries, Phys. Rev. B 75, 045417 (2007).

[42] C. W. J. Beenakker, Andreev reflection and Klein tunneling in graphene, Rev. Mod. Phys. 80, 1337 (2008).

[43] K. Komatsu, C. Li, S. Autier-Laurent, H. Bouchiat, and S. Guéron, Superconducting proximity effect in long superconductor/graphene/superconductor junctions: From specular Andreev reflection at zero field to the quantum Hall regime, Phys. Rev. B 86, 115412 (2012).

[44] D. K. Efetov, L. Wang, C. Handschin, K. B. Efetov, J. Shuang, R. Cava, T. Taniguchi, K. Watanabe, J. Hone, C. R. Dean, and P. Kim, Specular interband Andreev reflections at van der Waals interfaces between graphene and $\mathrm{NbSe}_{2}$, Nat. Phys. 12, 328 (2016).

[45] D. K. Efetov and K. B. Efetov, Crossover from retro to specular Andreev reflections in bilayer graphene, Phys. Rev. B 94, 075403 (2016).

[46] D. Greenbaum, S. Das, G. Schwiete, and P. G. Silvestrov, Pure spin current in graphene normal-superconductor structures, Phys. Rev. B 75, 195437 (2007).

[47] J. Cayssol, Crossed Andreev Reflection in a Graphene Bipolar Transistor, Phys. Rev. Lett. 100, 147001 (2008).

[48] J. Linder, M. Zareyan, and A. Sudbø, Spin-switch effect from crossed Andreev reflection in superconducting graphene spin valves, Phys. Rev. B 80, 014513 (2009).
[49] L. Wang, I. Meric, P. Y. Huang, Q. Gao, Y. Gao, H. Tran, T. Taniguchi, K. Watanabe, L. M. Campos, D. A. Muller, J. Guo, P. Kim, J. Hone, K. L. Shepard, and C. R. Dean, Onedimensional electrical contact to a two-dimensional material, Science 342, 614 (2013).

[50] V. E. Calado, S. Goswami, G. Nanda, M. Diez, A. R. Akhmerov, K. Watanabe, T. Taniguchi, T. M. Klapwijk, and L. M. K. Vandersypen, Ballistic Josephson junctions in edge-contacted graphene, Nat. Nanotechnol. 10, 761 (2015).

[51] M. Ben Shalom, M. J. Zhu, V. I. Fal'ko, A. Mishchenko, A. V. Kretinin, K. S. Novoselov, C. R. Woods, K. Watanabe, T. Taniguchi, A. K. Geim, and J. R. Prance, Quantum oscillations of the critical current and high-field superconducting proximity in ballistic graphene, Nat. Phys. 12, 318 (2016).

[52] F. Amet, C. T. Ke, I. V. Borzenets, J. Wang, K. Watanabe, T. Taniguchi, R. S. Deacon, M. Yamamoto, Y. Bomze, S. Tarucha, and G. Finkelstein, Supercurrent in the quantum Hall regime, Science 352, 966 (2016).

[53] I. V. Borzenets, F. Amet, C. T. Ke, A. W. Draelos, M. T. Wei, A. Seredinski, K. Watanabe, T. Taniguchi, Y. Bomze, M. Yamamoto, S. Tarucha, and G. Finkelstein, Ballistic Graphene Josephson Junctions from the Short to the Long Junction Regimes, Phys. Rev. Lett. 117, 237002 (2016).

[54] R. Kraft, J. Mohrmann, R. Du, P. B. Selvasundaram, M. Irfan, U. N. Kanilmaz, F. Wu, D. Beckmann, H. von Löhneysen, R. Krupke, A. Akhmerov, I. Gornyi, and R. Danneau, Tailoring supercurrent confinement in graphene bilayer weak links, Nat. Commun. 9, 1722 (2018).

[55] F. E. Schmidt, M. D. Jenkins, K. Watanabe, T. Taniguchi, and G. A. Steele, A ballistic graphene superconducting microwave circuit, Nat. Commun. 9, 4069 (2018).

[56] A. W. Draelos, M.-T. Wei, A. Seredinski, H. Li, Y. Mehta, K. Watanabe, T. Taniguchi, I. V. Borzenets, F. Amet, and G. Finkelstein, Supercurrent flow in multiterminal graphene Josephson junctions, Nano Lett. 19, 1039 (2019).

[57] P. Pandey, R. Kraft, R. Krupke, D. Beckmann, and R. Danneau, Andreev reflection in ballistic normal metal/ graphene/superconductor junctions, Phys. Rev. B 100, 165416 (2019).

[58] K.-F. Huang, Y. Ronen, R. Mélin, D. Feinberg, K. Watanabe, T. Taniguchi, and P. Kim, Interference of Cooper quartet Andreev bound states in a multi-terminal graphenebased Josephson junction, arXiv:2008.03419.

[59] G.-H. Park, K. Watanabe, T. Taniguchi, G.-H. Lee, and H.-J. Lee, Engineering crossed Andreev reflection in doublebilayer graphene, Nano Lett. 19, 9002 (2019).

[60] X. Wu, H. Meng, F. Kong, H. Zhang, Y. Bai, and N. Xu, Tunable nonlocal valley-entangled Cooper pair splitter realized in bilayer-graphene van der Waals spin valves, Phys. Rev. B 101, 125406 (2020).

[61] G. E. Blonder, M. Tinkham, and T. M. Klapwijk, Transition from metallic to tunneling regimes in superconducting microconstrictions: Excess current, charge imbalance, and supercurrent conversion, Phys. Rev. B 25, 4515 (1982).

[62] M. Octavio, M. Tinkham, G. E. Blonder, and T. M. Klapwijk, Subharmonic energy-gap structure in superconducting constrictions, Phys. Rev. B 27, 6739 (1983). 
[63] A. A. Golubov and M. Yu. Kupriyanov, Quasiparticle current of ballistic NcS'S contacts, JETP Lett. 61, 851 (1995), http:// www.jetpletters.ac.ru/ps/1209/article_18278.shtml.

[64] F. Pérez-Willard, J. C. Cuevas, C. Sürgers, P. Pfundstein, J. Kopu, M. Eschrig, and H. von Löhneysen, Determining the current polarization in $\mathrm{Al} / \mathrm{Co}$ nanostructured point contacts, Phys. Rev. B 69, 140502(R) (2004).

[65] See Supplemental Material at http://link.aps.org/ supplemental/10.1103/PhysRevLett.126.147701 for a full description of the sample fabrication, the analysis of the checkerboard pattern due to FP interferences, and a full description of our beam splitter model and the gate and temperature dependence of the CPS.

[66] It is to be noted that, while the superconducting terminals are in the normal state at this temperature, the corresponding junction is still called as the SGS junction for the sake of clarity.

[67] G. Giovannetti, P. A. Khomyakov, G. Brocks, V. M. Karpan, J. van den Brink, and P. J. Kelly, Doping Graphene with Metal Contacts, Phys. Rev. Lett. 101, 026803 (2008).

[68] P. A. Khomyakov, G. Giovannetti, P. C. Rusu, G. Brocks, J. van den Brink, and P. J. Kelly, First-principles study of the interaction and charge transfer between graphene and metals, Phys. Rev. B 79, 195425 (2009).

[69] P. A. Khomyakov, A. A. Starikov, G. Brocks, and P. J. Kelly, Nonlinear screening of charges induced in graphene by metal contacts, Phys. Rev. B 82, 115437 (2010).

[70] It is to be noted that these barriers are expected to be very smooth, in contrast to the gate-defined potential barriers [79-81], and therefore, angle-dependent transmission due to the Klein tunneling in single layer graphene [42,73] is expected to modify the transmission in the NGN junction.

[71] M. Houzet and P. Samuelsson, Multiple Andreev reflections in hybrid multiterminal junctions, Phys. Rev. B 82, 060517 (R) (2010).
[72] M. P. Nowak, M. Wimmer, and A. R. Akhmerov, Supercurrent carried by nonequilibrium quasiparticles in a multiterminal Josephson junction, Phys. Rev. B 99, 075416 (2019).

[73] M. I. Katsnelson, K. S. Novoselov, and A. K. Geim, Chiral tunnelling and the Klein paradox in graphene, Nat. Phys. 2, 620 (2006).

[74] G.-H. Lee, G.-H. Park, and H.-J. Lee, Observation of negative refraction of Dirac fermions in graphene, Nat. Phys. 11, 925 (2015).

[75] P. Rickhaus, P. Makk, M.-H. Liu, K. Richter, and C. Schönenberger, Gate tuneable beamsplitter in ballistic graphene, Appl. Phys. Lett. 107, 251901 (2015).

[76] D. R. da Costa, A. Chaves, S. H. R. Sena, G. A. Farias, and F.M. Peeters, Valley filtering using electrostatic potentials in bilayer graphene, Phys. Rev. B 92, 045417 (2015).

[77] R. Kraft, I. V. Krainov, V. Gall, A. P. Dmitriev, R. Krupke, I. V. Gornyi, and R. Danneau, Valley Subband Splitting in Bilayer Graphene Quantum Point Contacts, Phys. Rev. Lett. 121, 257703 (2018).

[78] H. Chen, P. Zhou, J. Liu, J. Qiao, B. Oezyilmaz, and J. Martin, Gate controlled valley polarizer in bilayer graphene, Nat. Commun. 11, 1202 (2020).

[79] A. F. Young and P. Kim, Quantum interference and Klein tunnelling in graphene heterojunctions, Nat. Phys. 5, 222 (2009).

[80] A. Varlet, M.-H. Liu, V. Krueckl, D. Bischoff, P. Simonet, K. Watanabe, T. Taniguchi, K. Richter, K. Ensslin, and T. Ihn, Fabry-Pérot Interference in Gapped Bilayer Graphene with Broken Anti-Klein Tunneling, Phys. Rev. Lett. 113, 116601 (2014).

[81] R. Du, M.-H. Liu, J. Mohrmann, F. Wu, R. Krupke, H. von Löhneysen, K. Richter, and R. Danneau, Tuning Anti-Klein to Klein Tunneling in Bilayer Graphene, Phys. Rev. Lett. 121, 127706 (2018). 